\title{
Turbulent Transition Mechanisms of Natural Convection over Upward-Facing Horizontal Plates*
}

\author{
Kenzo KITAMURA**, Xiao-An CHEN** \\ and Fumiyoshi KIMURA***
}

\begin{abstract}
Turbulent transition mechanisms and heat transfer characteristics of the natural convection over heated, horizontal plate were investigated both experimentally and analytically. An unsteady, 3-D numerical analysis has been performed on the water flow over $150 \mathrm{~mm}$-wide plate heated with constant heat flux. The analytical results showed that longitudinal vortices play a crucial role on the turbulent transition over the plate. The vortices appear first in the laminar boundary layer at certain distance from the leading edge. Then, they detach from the plate and become distorted toward downstream. Meanwhile, the flow and temperature fields over the plate were visualized experimentally. The results confirmed that the above transition actually occurs over the plate. Moreover, the local heat transfer coefficients predicted by the analysis coincide well with those measured by the experiments.
\end{abstract}

Key Words: Heat Transfer, Natural Convection, Transition to Turbulence, Visualization, Longitudinal Vortices, Three-Dimensional Analysis, Horizontal Plate

\section{Introduction}

Natural convective flows induced over heated, horizontal plates have been the subjects of numerous investigations during past decades. This is mainly because that the flows are encountered in a wide variety of engineering situations and of industrial equipment. A number of studies, therefore, have been carried out to investigate their flow and heat transfer characteristics both experimentally and analytically. However, the flows over heated, horizontal plates are potentially unstable because of top-heavy and bottomlight fluid situations. Thus, an exchange of the fluids occurs over the plates, resulting in complex flow and heat transfer characteristics of the natural convection. This also makes the analytical and experimental treatments difficult. Therefore, the flow and tempera-

* Received 3rd October, 2000. Japanese original: Trans. Jpn. Soc. Mech. Eng., Vol.65, No.640, B (1999), pp. 4033-4040 (Received 6th April, 1999)

** Department of Mechanical Engineering, Toyohashi University of Technology, 1-1 Hibarigaoka, Tempaku-cho, Toyohashi 441-8580, Japan. E-mail: kitamura@mech.tut.ac.jp

*** Faculty of Engineering, Himeji Institute of Technology, 2167 Shosha, Himeji 671-2201, Japan ture fields over horizontal plates are not completely understood when, in particular, compared with those over the vertical plates.

Considering the above, the present authors have carried out the intensive visualization experiments and heat transfer measurements on the natural convective flows of water over horizontal plates heated with uniform heat fluxes ${ }^{(1)-(3)}$. The experiments have covered the extremely wide range of the Rayleigh numbers, $R a_{w}^{*}=10^{6}-10^{15}$ by varying the plate width from $25 \mathrm{~mm}$ to $1500 \mathrm{~mm}$. On the course of the visualization experiments, we found specific flow and temperature-patterns appear over the plate. The typical examples are shown in Figs. 1(a) and 1(b), where the flow field and the surface temperatures of the plate $150 \mathrm{~mm}^{-}$-wide are visualized with dye and liquid crystal sheet, respectively. As is apparent from Fig. 1(a), the dye issued from the left edge of the plate first flows along the surface. Then, the dye concentrates and detaches three-dimensionally from the surface to become many filaments. These filaments are, then, distorted toward downstream and, finally, ascend away from the central portion of the plate as a turbulent plume. Although it is indistinct in the present black-and-white photo, one sees many streaky temperature-patterns in Fig. 1(b). These 


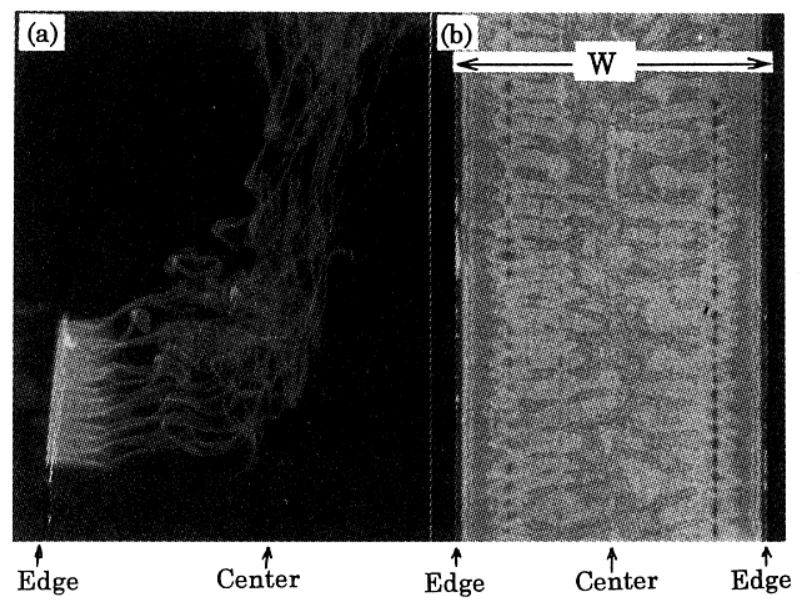

Fig. 1 Visualized flow field and surface temperature of $150 \mathrm{~mm}^{-}$wide plate

streaky patterns align with almost constant spanwise pitches and cover the considerable portion of the surface. Meanwhile, one can also see somewhat different, irregular-shaped temperature-patterns appear near the central portion of the plate. Based on the above results and also on the results with larger plates, the present authors have confirmed that the three-dimensional separation of the laminar boundary layer becomes a trigger to the turbulent transition over horizontal plates.

Meanwhile, Sparrow and Husar ${ }^{(4)}$ have first carried out the visualization experiments on the natural convective flows over heated horizontal and inclined plates using electro-chemical techniques. They reported that the longitudinal vortices, of which axes are parallel to the flow direction, appear over the plate in advance of the turbulent transition. Their results have attracted considerable interests of others, because such vortices have not been previously observed in this flow configuration. A number of visualization experiments have, therefore, been carried out successively to obtain further information on these vortices. For instance, Chen and $\mathrm{Kim}^{(5)}$ have conducted the visual experiments on the air flows over isothermal plate using smoke and have measured the onset conditions of the vortices and their spanwise pitches.

These visual experiments will provide basic insights into the turbulent transition phenomena over horizontal plates. However, we can point out several subjects remained unclear concerning with the turbulent transition. The one of such subjects is the role of the longitudinal vortices on the turbulent transition. We already know that the longitudinal vortices appear over the plate when the flows undergo a turbulent transition. However, it is not clear how these vortices are related to the turbulent transition. The another subject is concerned with the transition pro- cess. The comprehensive information on the transition process from the first beginning of transition to the fully turbulent state is not available at present. Moreover, the transition of flow will exert significant influences on the local heat transfer from the plate. However, very little information has been available on the heat transfer characteristics, in particular, in the transitional region.

In order to investigate these subjects, analytical simulations of the turbulent transition process will be necessary, because the visual experiments can afford limited information on the transition process. In light of this, we will next review the previous analytical studies. There were several analytical studies that have dealt with the natural convection over horizontal plate. However, main concerns of these studies have been directed to the laminar flows in the vicinity of the leading edges. For example, Pera and Gebhart ${ }^{(6)}$ and Chen and colleagues $^{(7)}$ have conducted the analysis using similarity parameters and have obtained the velocity and temperature distributions of the laminar boundary layer. Meanwhile, to the best of the authors' knowledge, two-dimensional numerical analysis by Ishiguro and colleagues ${ }^{(8)}$ was the only work that have dealt with high Rayleigh number flows. They reported that oscillating flows take place over the plate when Rayleigh numbers exceed certain critical value. However, the previous experiments by the authors revealed that the above oscillating flows are not concerned directly with the turbulent transition phenomena.

The above circumstances motivate the present numerical analysis. The analysis aims to simulate the whole transition process including the generation of the longitudinal vortices, the separation of laminar boundary layer flow, and the successive distortion of flows. The analytical results are, then, compared to the experimental results to ascertain the availability of the present analysis. The present analysis will provide comprehensive information on the turbulent transition mechanisms over horizontal plates. Moreover, the results will be of great help to investigate the turbulent transition mechanisms of the natural convections over upward-facing, inclined plates and around horizontal cylinders. Because, similar transition phenomena as above have been also observed in those flows ${ }^{(9),(10)}$.

\section{Analysis}

As is apparent from the previous visual results, the turbulent transition phenomena over horizontal plate are potentially three-dimensional and timedependent. Thus, we have adopted an unsteady, threedimensional analysis to simulate the transition process. 
Figure 2 shows the analytical coordinate system utilized in the present analysis. The analysis considers a horizontal plate having finite width, $W$ and span, $S$, which is heated with a constant heat flux, $q_{w}$. The plate was flush mounted to the bottom of a solution domain of width, $(W / 2+L)$, height, $H$ and Span, $S$. We also adopted the Cartesian coordinate system $x, y$, and $z$ and the corresponding velocity $u$, $v$ and $w$ as are shown in the figure. Assuming the constant properties of the fluid except for the buoyancy term of the $y$-momentum equation, the governing equations become as:

Conservation of mass

$$
\frac{\partial u}{\partial x}+\frac{\partial v}{\partial y}+\frac{\partial w}{\partial z}=0
$$

Conservation of momentum

$$
\begin{aligned}
& \rho\left(\frac{\partial u}{\partial \tau}+u \frac{\partial u}{\partial x}+v \frac{\partial u}{\partial y}+w \frac{\partial u}{\partial z}\right) \\
& \quad=-\frac{\partial p}{\partial x}+\mu\left(\frac{\partial^{2} u}{\partial x^{2}}+\frac{\partial^{2} u}{\partial y^{2}}+\frac{\partial^{2} u}{\partial z^{2}}\right) \\
& y \text {-momentum } \\
& \rho\left(\frac{\partial v}{\partial \tau}+u \frac{\partial v}{\partial x}+v \frac{\partial v}{\partial y}+w \frac{\partial v}{\partial z}\right) \\
& \quad=-\frac{\partial p}{\partial y}+\mu\left(\frac{\partial^{2} v}{\partial x^{2}}+\frac{\partial^{2} v}{\partial y^{2}}+\frac{\partial^{2} v}{\partial z^{2}}\right)+\left(\rho_{\infty}-\rho\right) g
\end{aligned}
$$

$$
z \text {-momentum }
$$

$$
\begin{aligned}
& \rho\left(\frac{\partial w}{\partial \tau}+u \frac{\partial w}{\partial x}+v \frac{\partial w}{\partial y}+w \frac{\partial w}{\partial z}\right) \\
& \quad=-\frac{\partial p}{\partial z}+\mu\left(\frac{\partial^{2} w}{\partial x^{2}}+\frac{\partial^{2} w}{\partial y^{2}}+\frac{\partial^{2} w}{\partial z^{2}}\right)
\end{aligned}
$$

Conservation of energy

$$
\begin{aligned}
& \rho C p\left(\frac{\partial T}{\partial \tau}+u \frac{\partial T}{\partial x}+v \frac{\partial T}{\partial y}+w \frac{\partial T}{\partial z}\right) \\
& =\lambda\left(\frac{\partial^{2} T}{\partial x^{2}}+\frac{\partial^{2} T}{\partial y^{2}}+\frac{\partial^{2} T}{\partial z^{2}}\right)
\end{aligned}
$$

In the present analysis, Equations (1) to (5) were discretized directly by using a control-volume

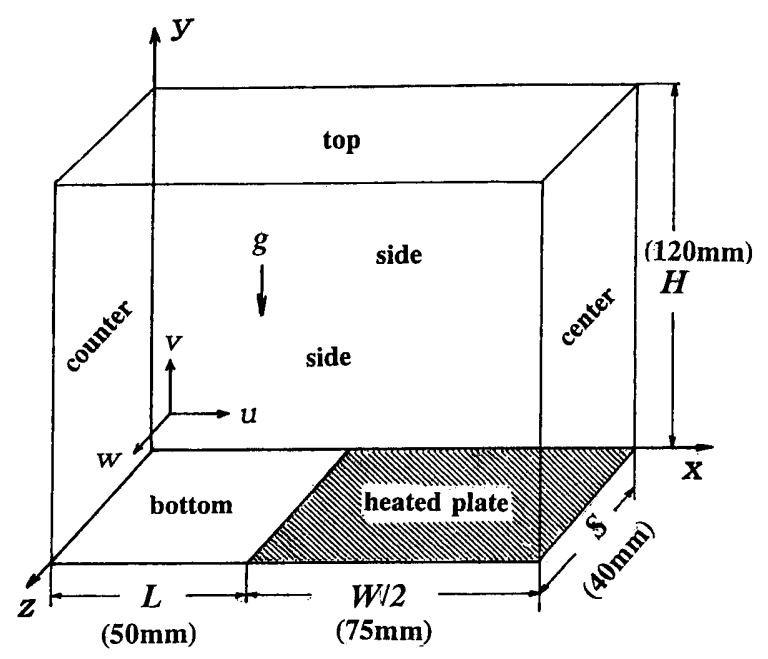

Fig. 2 Analytical co-ordinate system scheme. A third-order upwind difference and a secondorder central difference scheme were utilized to discretize the convection terms and the other terms, respectively. Moreover, a SMAC scheme, which solves the velocity field explicitly, while the pressure field implicitly, was used for the time-integration. A time increment $\Delta \tau$ was selected so as to satisfy the Courant condition and also the stability criteria for the viscosity and thermal diffusions.

To make the comparison with the experimental results easier, the present calculations were carried out for water at room temperature, $\mathrm{Pr}=6$. A $150 \mathrm{~mm}^{-}$ wide plate heated with uniform heat fluxes was also adopted as a test plate. The size was selected from the following reasons. The whole transition phenomena will be realized over the plate with the above size. While, the flow over plate will not be fully turbulent, so that the laminar equations from ( 1 ) to $(5)$ can be utilized for the prediction of the transition process.

In order to determine the appropriate sizes of the calculation domain, preliminary computations were made by varying the sizes of $L, H$ and $S$ in the ranges as $L=30-100 \mathrm{~mm}, H=50-200 \mathrm{~mm}$ and $S=20-60$ $\mathrm{mm}$. As a result, calculated heat-transfer coefficients of the plate converged within $\pm 3.2 \%$, when $L, H$ and $S$ are larger than $50 \mathrm{~mm}, 100 \mathrm{~mm}$ and $30 \mathrm{~mm}$, respectively. Taking account of this fact and also in view of the necessary computation time and memories, the values $L=50 \mathrm{~mm}, H=120 \mathrm{~mm}$ and $S=40 \mathrm{~mm}$ were adopted as the domain sizes. The span $S=40 \mathrm{~mm}$ was also selected from the experimental result that the spanwise pitches of the low-temperature streaks are less than $10 \mathrm{~mm}$. Thus, several streaks may appear within the above span and this will enable the accurate measurement of the spanwise pitches of the lowtemperature streaks as will be mentioned in the below. Meanwhile, making use of the symmetrical flow configuration over the horizontal plate, only half of the calculation domain was considered in the analysis, so that the calculation was proceeded in the region from the leading edge to the mid-plane of the test plate, $W / 2$.

The majority of computations were made using a $70 \times 40 \times 50$ (140000 total nodes) grid. In the $x$-direction, fifty grids of equal spacing were placed over the test plate, while twenty grids of unequal spacing were placed over the bottom plane (see Fig. 2 for plane name). In the $y$-direction, the grids distribute densely near the bottom boundary and sparsely near the top boundary. While, uniform grids were deployed in the $z$-direction. The grid dependence was checked by carrying out the computations with finer grid sizes than those of the above. We confirmed that the heat transfer coefficients changed by less than $\pm 2.5 \%$. 
Table 1 Boundary conditions

\begin{tabular}{lll}
\hline \multicolumn{1}{c}{ Plane $^{*}$} & For Velocity & For Temperature \\
\hline heated plate & $\mathrm{u}=\mathrm{v}=\mathrm{w}=0$ (non-slip) & $\mathrm{q}_{\mathrm{w}}=$ const(given) \\
bottom & $\partial(\mathrm{u}, \mathrm{v}, \mathrm{w}) / \partial \mathrm{y}=0$ & $\mathrm{~T}=\mathrm{T}_{\infty}$ \\
top & $\partial(\mathrm{u}, \mathrm{v}, \mathrm{w}) / \partial \mathrm{y}=0$ & $\partial \mathrm{T} / \partial \mathrm{y}=0$ \\
middle & $\mathrm{u}=0, \partial(\mathrm{v}, \mathrm{w}) / \partial \mathrm{x}=0$ & $\partial \mathrm{T} / \partial \mathrm{x}=0$ \\
counter & $\partial(\mathrm{u}, \mathrm{v}, \mathrm{w}) / \partial \mathrm{x}=0$ & $\mathrm{~T}=\mathrm{T}_{\infty}$ \\
side & $\mathrm{w}=0, \partial(\mathrm{u}, \mathrm{v}) / \partial \mathrm{z}=0$ & $\partial \mathrm{T} / \partial \mathrm{z}=0$ \\
\hline
\end{tabular}

* see Fig. 2 for reference

The boundary conditions at the computational domain are listed in Table 1. The initial conditions for velocities and temperature were imposed as : $u=$ $v=w=0$ and $T=T_{\infty}$, where $T_{\infty}$ was set as $T_{\infty}=20^{\circ} \mathrm{C}$ to coincide with the experimental condition. The properties of water were estimated at the film-temperature, $T_{f}=\left(T_{w}+T_{\infty}\right) / 2$ except for the density in the buoyancy term, which was calculated from the programmed data table. The computations were carried out with the surface-heat fluxes ranging as $q_{w}=1-7 \mathrm{~kW} / \mathrm{m}^{2}$. Based on the above plate width and heat fluxes, the modified Rayleigh numbers $R a_{w}^{*}(=$ $\left.g \beta q_{w} W^{4} / \lambda \nu \alpha\right)$ was ranged as : $R a_{w}^{*}=1.5 \times 10^{10}-1.1 \times 10^{11}$.

In the present analysis, the time-dependent computations were made under the prescribed boundary and initial conditions. The results showed that the surface temperatures in the laminar region converge to constant values with the time, while the temperatures in the downstream region of the separation still fluctuate irregularly even if the computation time is large enough. Taking account of the above fact, we defined a pseudo-steady state with the state that the temperatures in the laminar region converged to constant values. We also confirmed that the pseudosteady state is accomplished within the time period of 300 seconds after the heating starts. The analytical results mentioned in the below are all concerned with this pseudo-steady state.

\section{Results and Discussion}

\section{1 Flow and temperature fields over horizontal plate}

We will first mention the analytical results on the flow and temperature fields over horizontal plate. Figures 3 and 4 show representative results of the velocity vectors and isotherms in the $y-z$ plane at different location of $X$, while the time and the surface heat flux are kept constant as : $\tau=350 \mathrm{sec}$. and $q_{w}=$ $3 \mathrm{~kW} / \mathrm{m}^{2}$. In the cross-section of $X=6 \mathrm{~mm}$, the fluid temperatures are uniform in the spanwise direction, and no specific flow is observed over the plate. Meanwhile, the temperatures begin to wave in the spanwise direction and small vortices appear over the plate at $X=10.5 \mathrm{~mm}$. The above waves and vortices are amplified at $X=13.5 \mathrm{~mm}$. Then, they are aligned

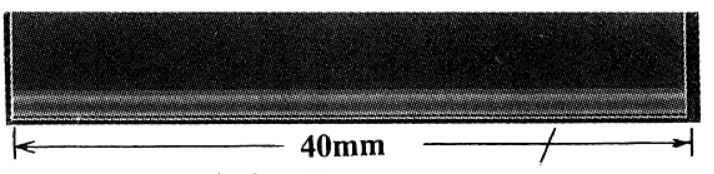

(a) $X=6 \mathrm{~mm}$ heated plate

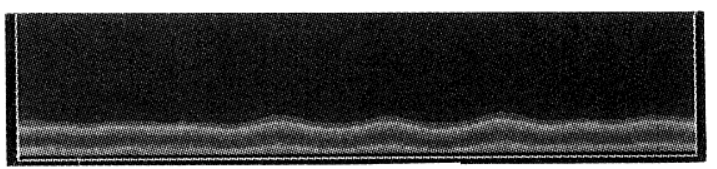

(b) $X=10.5 \mathrm{~mm}$

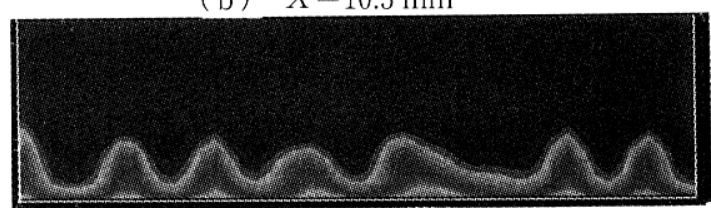

(c) $X=13.5 \mathrm{~mm}$

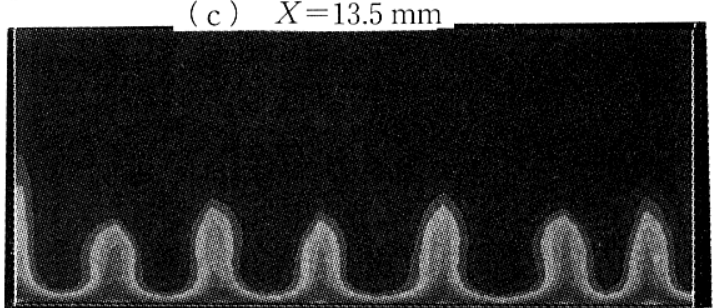

(d) $X=30 \mathrm{~mm}$

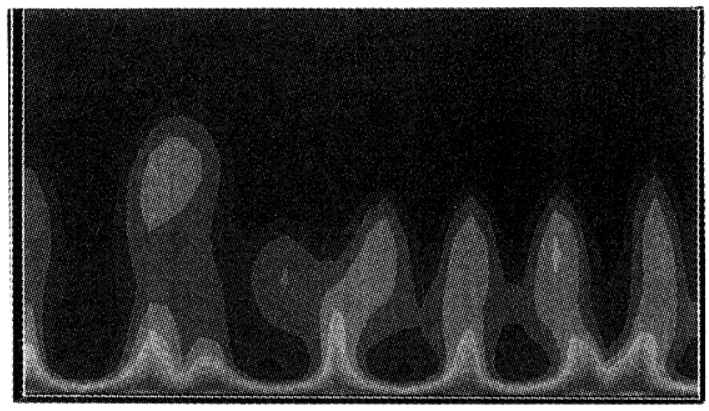

(e ) $X=45 \mathrm{~mm}$

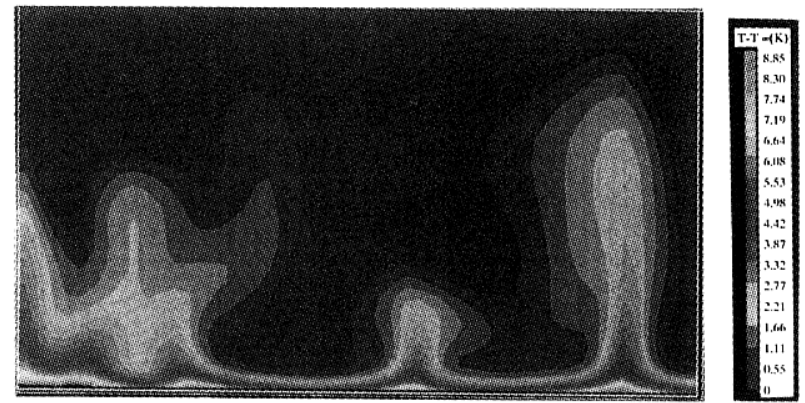

(f) $X=60 \mathrm{~mm}$

Fig. 3 Isotherms in $y-z$ plane $\left(q_{w}=3000 \mathrm{~W} / \mathrm{m}^{2}\right.$, $\tau=350 \mathrm{sec}$ )

with almost constant spanwise pitches in the plane of $X=30 \mathrm{~mm}$. Figures $3(\mathrm{~d})$ and $4(\mathrm{~d})$ also depict that the lift-up of high-temperature fluids occurs at the portion of upward velocity vectors, while that the portions of low-temperatures correspond to those of downward velocity vectors. With further increase in $X$, the waves and vortices begin to breakup and a part of them detach from the surface as is shown in Figs. 


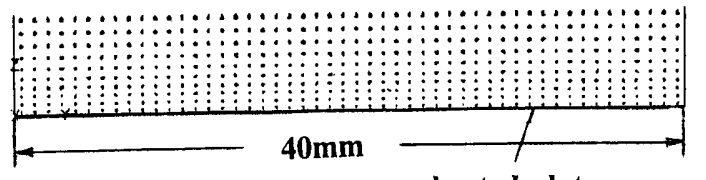

(a) $X=6 \mathrm{~mm}$
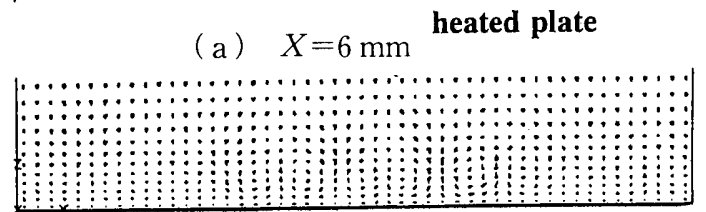

(b) $X=10.5 \mathrm{~mm}$

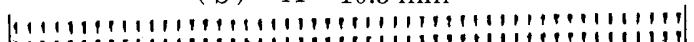

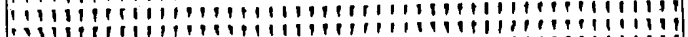

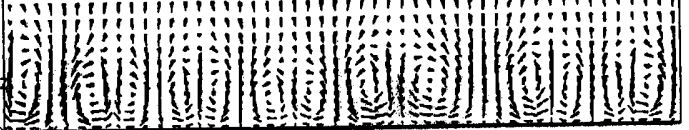

(c) $X=13.5 \mathrm{~mm}$

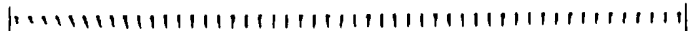

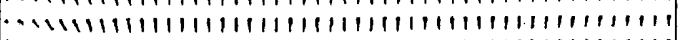

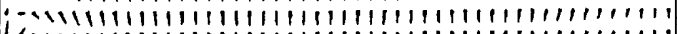

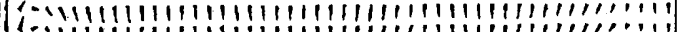

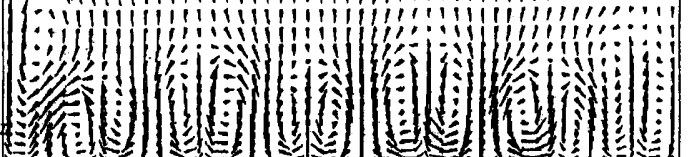
(d) $X=30 \mathrm{~mm}$

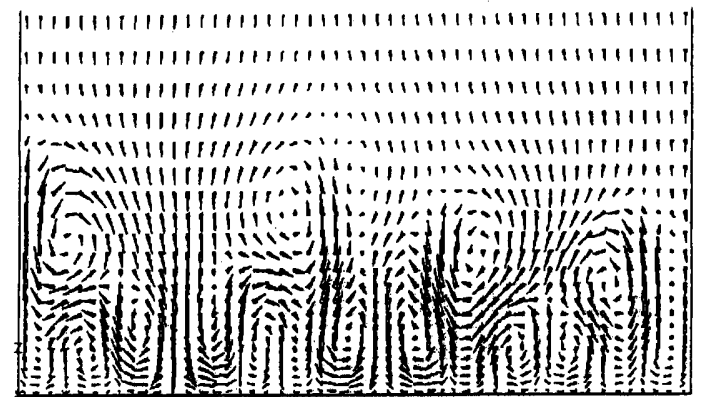

(e) $X=45 \mathrm{~mm}$

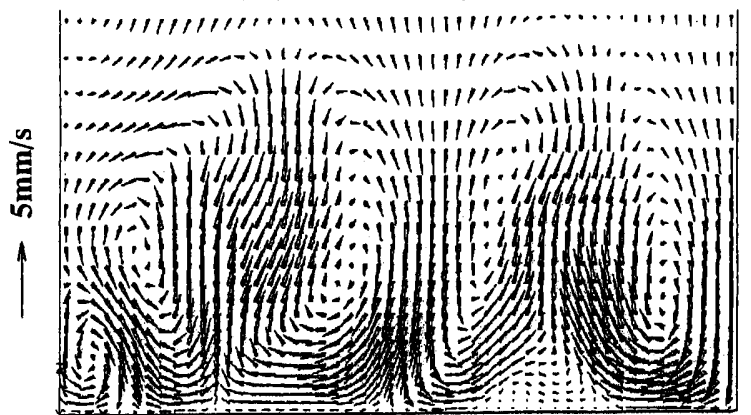

(f) $X=60 \mathrm{~mm}$

Fig. 4 Velocity vectors in $y-z$ plane $\left(q_{w}=3000 \mathrm{~W} / \mathrm{m}^{2}\right.$, $\tau=350 \mathrm{sec}$ )

3(e) and 4 (e ). Finally, the temperature and velocity fields over the plate are distorted significantly in the downstream section of $X=60 \mathrm{~mm}$, where irregularshaped, large vortices appear over the plate in Fig. 4 (f). Figures 3 and 4 represent the instantaneous isotherms and velocity vectors at $\tau=350 \mathrm{sec}$. While, the results at different time steps demonstrated that the waves and vortices change their location and shapes irregularly with time.
The above results also confirmed that the turbulent transition undergoes through the following process. Firstly, unstable stratification of the fluids occurs over the plate. Then, the vertical exchange of fluids takes place to dissolve the above instability. This also results in the longitudinal-vortices. These vortices are, then, distorted toward downstream, and finally, a fully turbulent state is realized over the plate.

In order to assure the above analytical results, we have next conducted the visualization experiments under the same conditions as above and the results were compared with the analytical results. Figure 5 shows the typical results of the visualized flow fields in the identical planes as those of Figs. 3 and 4. Here, the dye was issued from the long slit attached to the leading edge of the plate, and the cross sectional movement of the dye was made visible by using a laser-sheet. The temperature of the dye will become higher through flowing over the heated plate, and, thus, the dye is supposed to represent the movement of the high-temperature fluid. As is obvious from Fig. 5 (a), the dye spreads in the vicinity of the plate indicating that the laminar boundary layer flow is realized in the cross section of $X=6 \mathrm{~mm}$. Then, the dye begins to wave in the spanwise direction at $X=10.5$ $\mathrm{mm}$ and grows to be a mushroom-shape as shown in Fig. 5(c). One sees the legs of the mushrooms detach from the plate in Fig. $5(\mathrm{~d})$. This is observed as the separation of dye shown in Fig. 1(a). While, the detached dyes are distorted markedly in the plane $X=45 \mathrm{~mm}$ and ascend away from the plate as shown in Fig. 5( f ). Although we cannot compare these visual results directly with the analytical results in Figs. 3 and 4 , the present analytical results well predict the movement of the high-temperature fluids.

For further confirmation of the present analysis, the flow field was also visualized with fine particles and the result was compared with the analytical velocity vectors. Figure 6 shows an example of such comparison, where the visualized and computed velocity fields in the cross-section of $X=13.5 \mathrm{~mm}$ are represented with the identical scale. The visual result in Fig. 6(b) depicts the occurrence of counter-rotating, vortex pairs in the vicinity of the plate. Similar vortex pairs are also predicted by the analysis as shown in Fig. 6(a). Moreover, these vortices are of the same scales in between the analysis and experiment.

From the above comparisons, it is expected that the present analysis well simulate the actual flow and temperature fields. However, the above comparisons are qualitative rather than quantitative. Thus, further comparisons were made on the surface temperatures of the test plate in the next. 

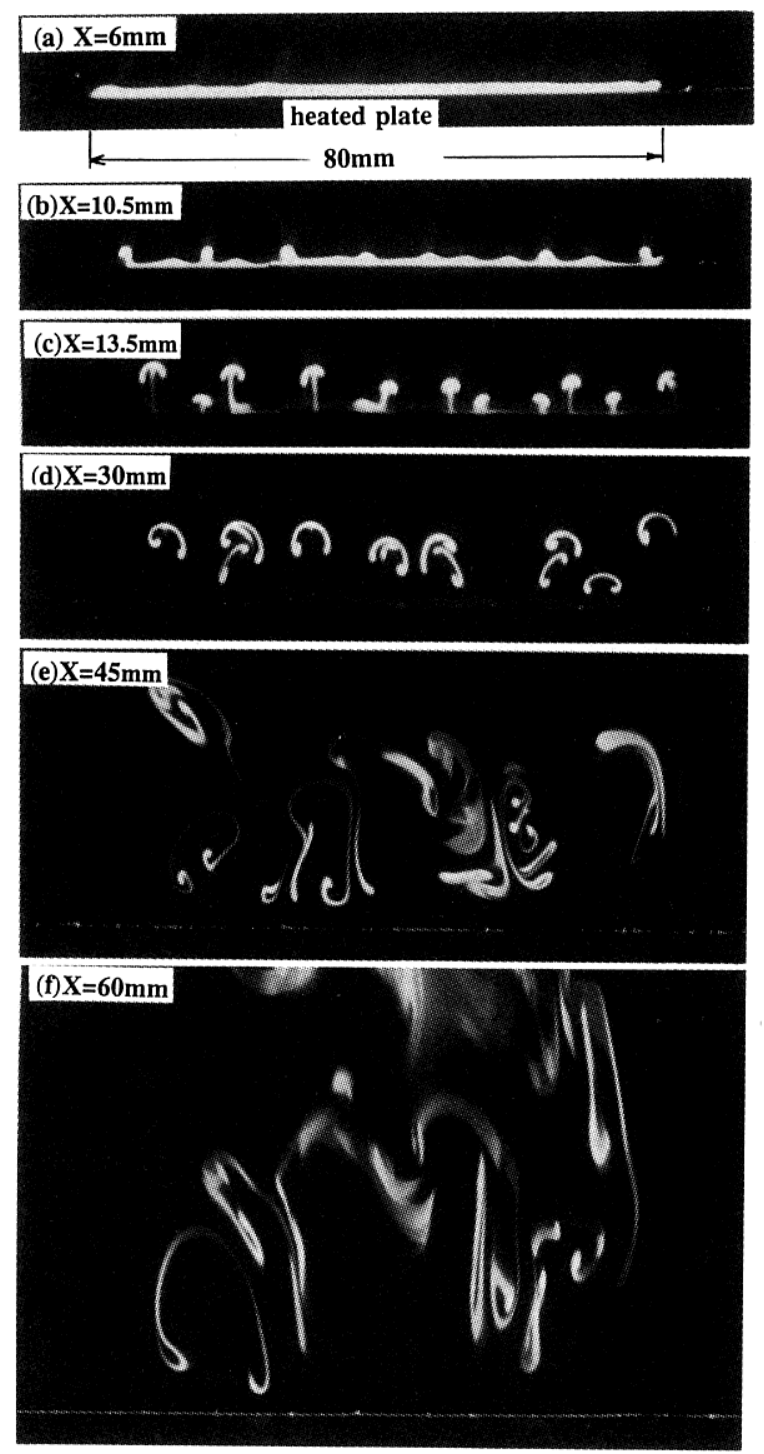

Fig. 5 Visualized flow field in $y-z$ plane $\left(q_{w}=3000 \mathrm{~W} / \mathrm{m}^{2}\right)$

\section{2 Surface temperatures of the test plate}

As was mentioned previously, surface temperatures of the constant-flux plate show specific patterns. If these patterns are predicted also by the analysis, the result will become one of the convincing proofs for the present analysis. Considering this, the present authors have drawn the contour maps of the surface temperatures based on the computed results and the results have been compared with the visualized temperature patterns. The analytical and experimental results are presented in Figs. 7 and 8 with the cases of $q_{w}=1,3$ and $5 \mathrm{~kW} / \mathrm{m}^{2}$. Although, the original color contours are indistinct in these black-and-white figures, the following temperature patterns appear commonly in the both figures. Those are; (i ) two-dimensional pattern near the leading edge, where temperatures are uniform in the spanwise direction, while they increase rapidly toward downstream, (ii ) streaky, low-temperature patterns that appear at certain distance from

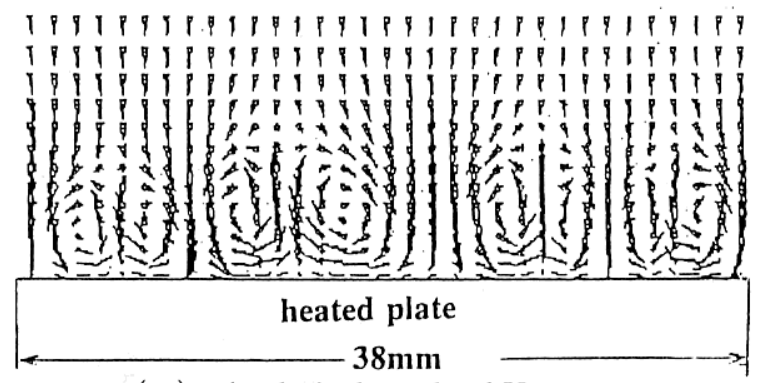

(a) Analytical result of Vector

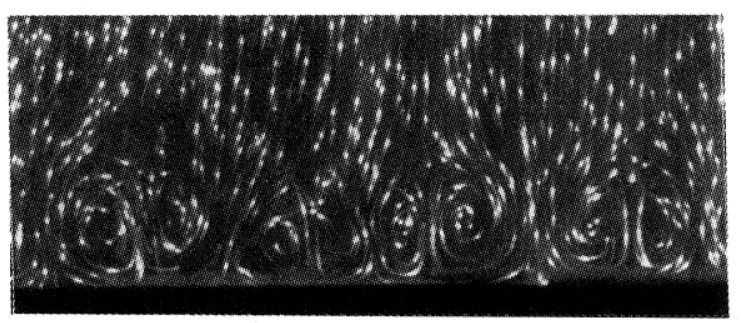

(b) Visualized trajectories of particles

Fig. 6 Comparison of flow field at $X=13.5 \mathrm{~mm}$

the edge, and (iii) irregular-shaped, low-temperature patterns that appear near the center of the plate. Moreover, similar variations with the surface heat flux are obvious for the temperature patterns (ii). Namely, the onset of the low-temperature streaks shifts toward downstream and their spanwise pitches become smaller with the surface heat fluxes. Meanwhile, the onset point and pitch of these streaks will become a good measure to ascertain the availability of the present analysis. Thus, we subsequently measured these quantities both from the analytical and experimental results.

As is shown in Figs. 7 and 8, the onset points and the pitches of the streaks vary significantly with each streak. Besides, they vary with a time period of several to ten seconds. Thus, we defined the onset point and the pitch with the values averaged with respect to time and space. On the measurements of the time-average values from the experiment, the whole visual results were once stored with VCR, and their still pictures were reproduced with constant period of 10 seconds to measure the spatial averaged values. Then, these measurements were repeated at least fifty times to yield the time-averaged values. Similar measurements as above were also performed on the analytical results, where the results at each period of 10 seconds were utilized to measure the timeaveraged values.

The onset points and pitches of the streaks thus obtained were given in Fig. 9, where the streamwise distances from the leading edge to the onset of streaks, $S$, and the spanwise pitches between streaks, $P$, are plotted with surface heat flux, $q_{w}$. The figure depicts that the distances $S$ obtained from the present 

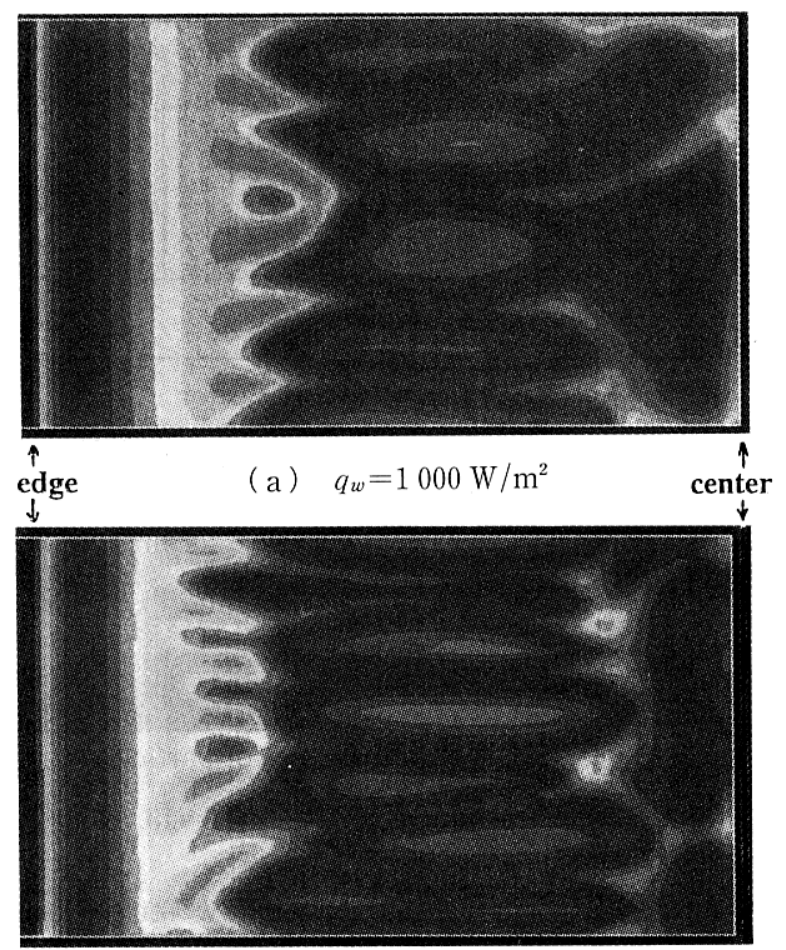

(b) $\quad q_{w}=3000 \mathrm{~W} / \mathrm{m}^{2}$

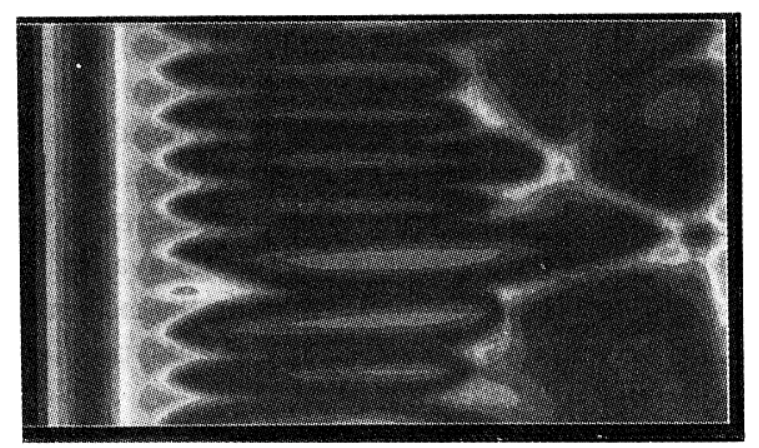

(c) $q_{w}=5000 \mathrm{~W} / \mathrm{m}^{2}$

Fig. 7 Analytical isotherms of surface temperatures

analysis agree well with the experimental distances. The values ranged from 10 to $20 \mathrm{~mm}$ and become smaller with the heat flux. A good coincidence is also apparent for the spanwise pitches $P$ in between the analysis and experiment. The results show monotonous decrease of $P$ from 9 to $4 \mathrm{~mm}$ with the increase in the heat flux from 1 to $6 \mathrm{~kW} / \mathrm{m}^{2}$.

For further information on these quantities, the distances $S$ and the pitches $P$ are normalized with the modified Rayleigh numbers as; $R a_{s}^{*}\left(=g \beta q_{w} S^{4} / \lambda \nu \alpha\right)$ and $R a_{p}^{*}\left(=g \beta q_{w} P^{4} / \lambda \nu \alpha\right)$. The results are presented in Fig. 10 in terms of the surface heat flux. The figure depicts that the Rayleigh numbers for the onsets and pitches become constant as $R a_{s}^{*}=3.5 \times 10^{6}$ and $R a_{p}^{*}=$ $1.46 \times 10^{5}$ regardless of the heat flux. Taking account of the fact that the onset of the streaks also corresponds to the first onset of turbulent transition, the former result, $R a_{s}^{*}=3.5 \times 10^{6}$, will give critical Rayleigh num-

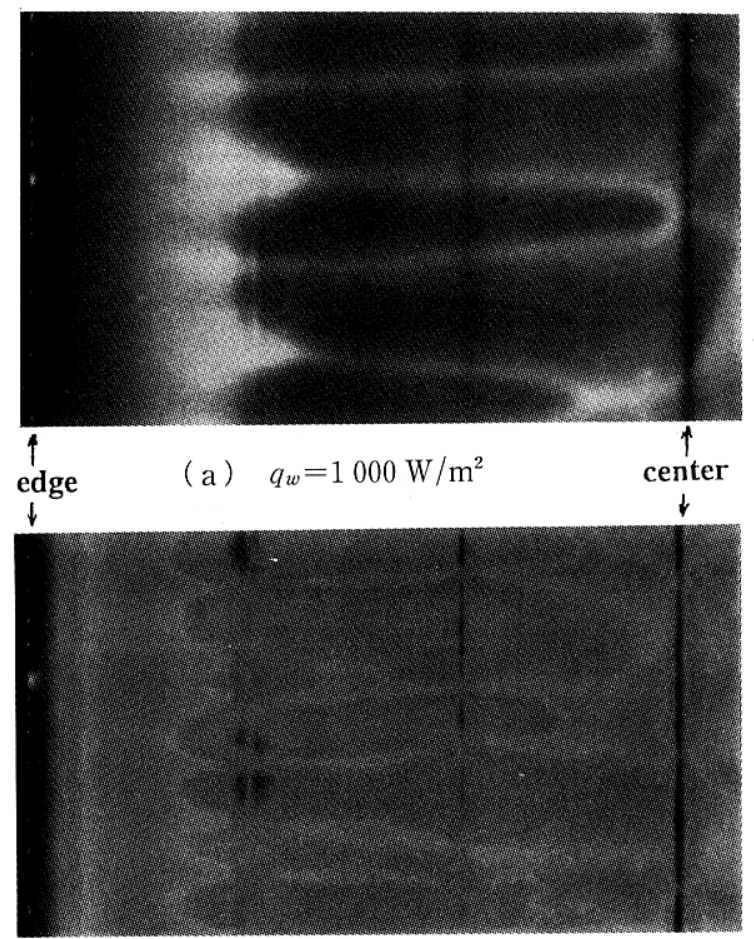

(b) $\quad q_{w}=3000 \mathrm{~W} / \mathrm{m}^{2}$

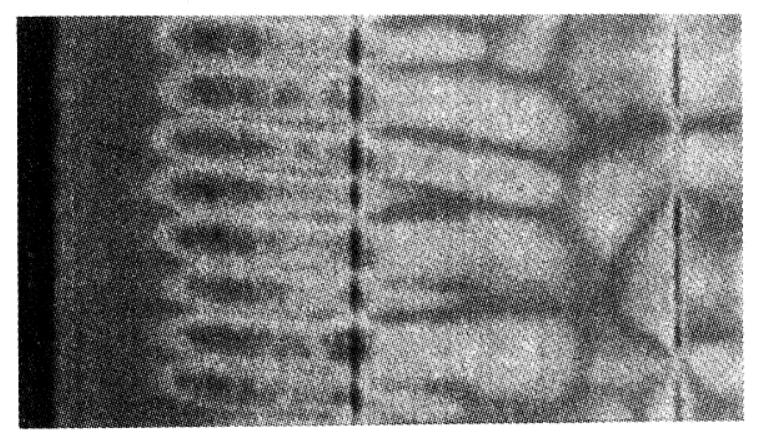

(c) $q_{w}=5000 \mathrm{~W} / \mathrm{m}^{2}$

Fig. 8 Visualized surface temperatures by liquid crystal

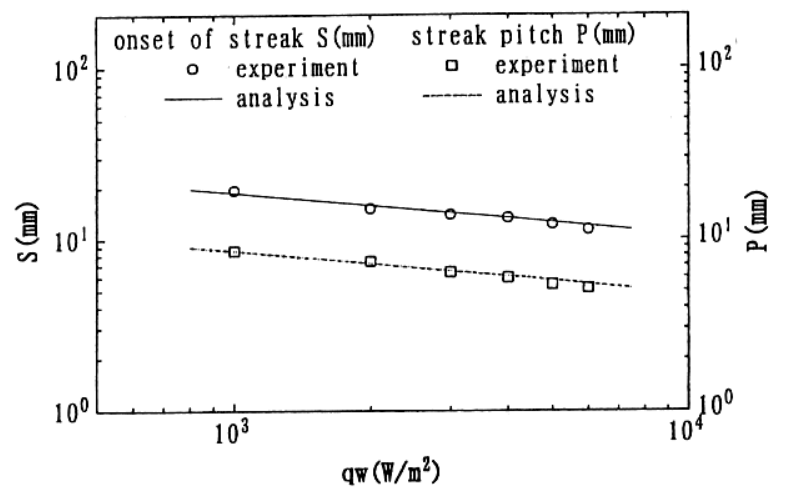

Fig. 9 Onset point and spanwise pitch of streaks

ber for the turbulent transition.

\section{3 Heat transfer characteristics}

The local heat transfer coefficients calculated from the present analysis were next compared with 
those from the experiment. The results are presented in Fig. 11, where the local coefficients, $h_{x}$ under the prescribed surface heat fluxes are plotted in terms of the streamwise distance $X$. The heat transfer coefficients are defined as:

$$
h_{x}=q_{w} /\left(T_{w x}-T_{\infty}\right)
$$

where, $T_{w x}$ and $T_{\infty}$ stand for the wall temperature at location $X$ and the ambient temperature of the fluid. Due to the occurrence of the low-temperature streaks, the wall temperatures in the transition region show marked variations in the spanwise direction. Thus, we defined $T_{w x}$ as the temperatures averaged spanwise. On the calculation of $T_{w x}$ from the analysis, the instantaneous temperatures were averaged first in the spanwise direction and, then, they are averaged again with time. Meanwhile in the experiment, the output signals of the thermocouples, which were spotwelded on the back of the foil heaters, were once recorded with a pen-recorder for a period of 5 minutes. Then, their signals were averaged with time to obtain $T_{w x}$. The ambient temperature of water, $T_{\infty}$, was measured with the thermocouple placed in the same horizontal plane as the plate and $150 \mathrm{~mm}$ apart from the plate edge.

As is apparent from the figure, the present analytical coefficients agree well with the experimental coefficients. The highest coefficients are achieved at the leading edge, and the coefficients decrease rapidly

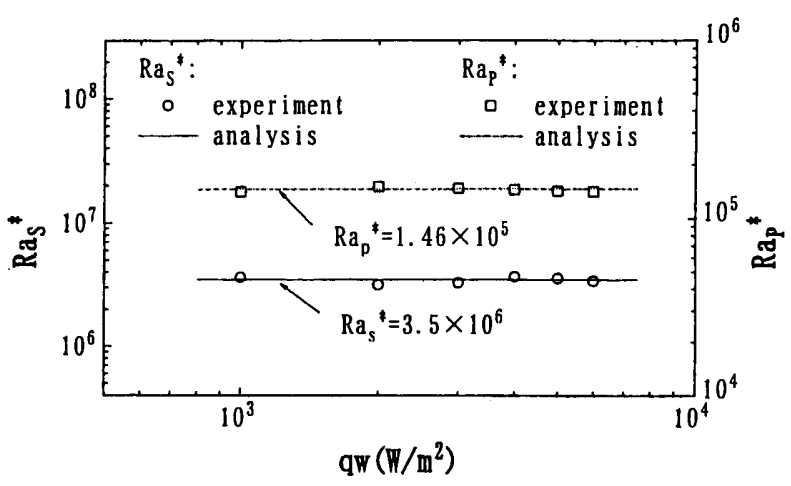

Fig. 10 Non-dimensional onset point and pitches with $X$ and reach minimums at certain distances $X$. Then, they turn to increase and show maximums. In the downstream region of the maximums, they gradually decrease with $X$. It is also obvious that the maximum and minimum points gradually shift toward upstream with the heat flux. Comparing these coefficients with the visual results in Figs. 7 and 8, the minimum points were found to occur at the location just upstream of the low-temperature streaks, while the maximum coefficients were realized at the location where the streaks expand widest in the spanwise direction.

For further discussion on these coefficients, they were replotted in $N u_{x}-R a_{x}^{*}$ plane as shown in Fig. 12, where $N u_{x}$ and $R a_{x}^{*}$ stand for the local Nusselt numbers and the local modified Rayleigh numbers expressed as: $N u_{x}=h_{x} X / \lambda$ and $R a_{x}^{*}=g \beta q_{w} X^{4} / \lambda \nu \alpha$. The analytical Nusselt numbers show linear variations against the Rayleigh numbers, which are shown with the solid lines in the figure. Moreover, the experimental Nusselt numbers gather around these lines indicating the coincidence between the analysis and experiment.

Assuming the correlation between the Nusselt and Rayleigh numbers with the form: $N u_{x}=C R a_{x}^{* n}$, we next calculated the coefficient $C$ and the exponent $n$ from the figure. Then, the results become as:

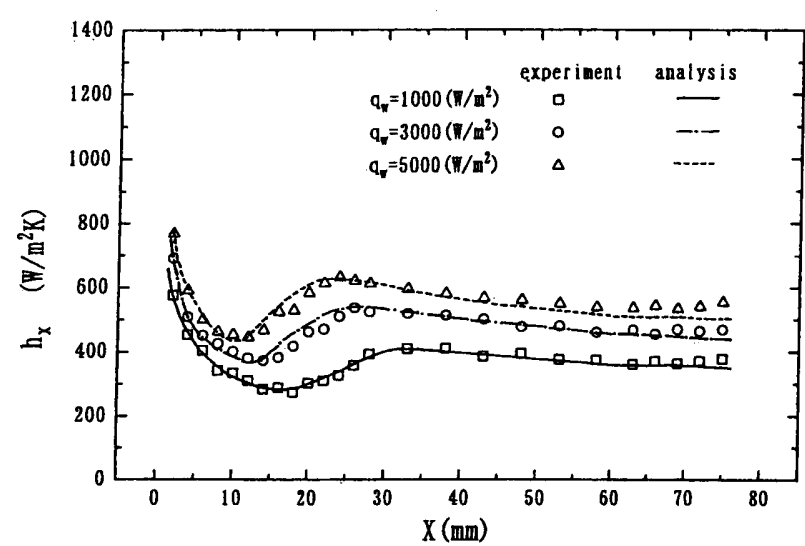

Fig. 11 Local heat transfer coefficients

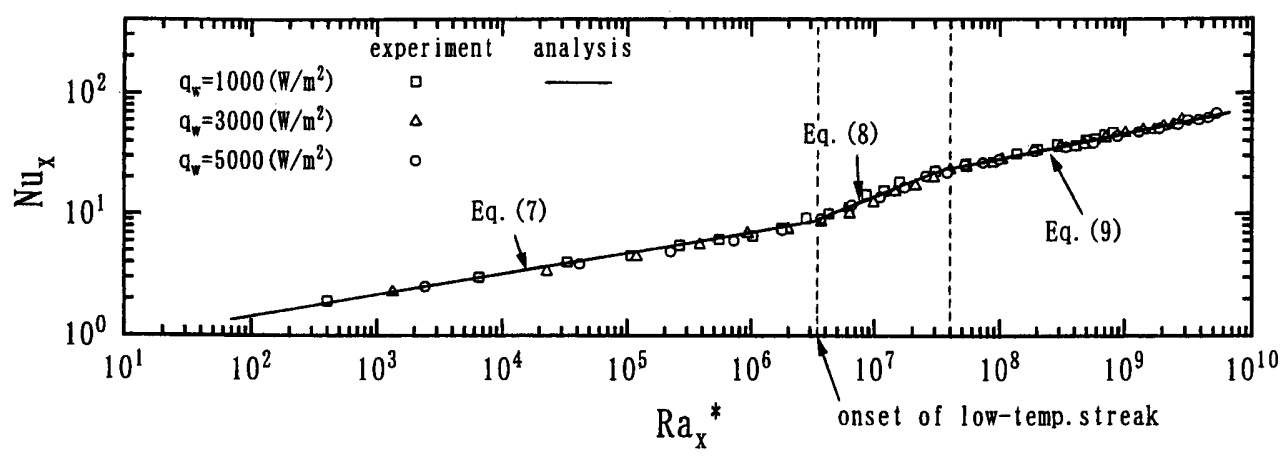

Fig. 12 Local Nusselt numbers of the plate 


$$
\begin{aligned}
& \text { (i ) } R a_{x}^{*}<3.5 \times 10^{6} \\
& N u_{x}=0.66 R a_{x}^{* 1 / 6} \\
& \text { (ii) } 3.5 \times 10^{6}<R a_{x}^{*}<4 \times 10^{7} \\
& N u_{x}=0.0125 R a_{x}^{* 0.43} \\
& \text { (iii) } \quad 4 \times 10^{7}<R a_{x}^{*}<8 \times 10^{9} \\
& N u_{x}=0.70 R a_{x}^{* 1 / 5}
\end{aligned}
$$

According to the previous experimental results by the authors, the above Eq. ( 9 ) can be extrapolated to the higher Rayleigh numbers, $R a_{x}^{*}=8 \times 10^{10}$. Moreover, the previous results also depicted that the following turbulent correlation is applicable in the region of higher Rayleigh numbers, $8 \times 10^{10}<R a_{x}^{*}<10^{14}$.

$$
N u_{x}=0.20 R a_{x}^{* 1 / 4}
$$

Based on the latter result, the present analytical range still remains in the transitional region. Meanwhile, it is worth while investigation whether the present analysis is applicable or not to the flows of higher Rayleigh numbers. Thus, we are planning to conduct the similar analysis in the turbulent region in the future.

\section{Conclusions}

The turbulent transition mechanisms of the natural convection over upward-facing horizontal plate were investigated both analytically and experimentally in the present study. The unsteady, three-dimensional analysis has been carried out to simulate the transition phenomena and the results were compared with the experimental results. Water at room temperature and $150 \mathrm{~mm}^{-}$wide plate heated with constant heat flux was adopted as a test fluid and a test plate. The following results were obtained from the present analysis and experiment.

(1) The analytical results show that the flow over horizontal plate undergoes a turbulent transition through the following process. (i) The laminar boundary layer flow first appears in the vicinity of the plate edge. (ii) The flow gradually becomes unstable due to heating. To dissolve the above instability, the longitudinal vortices take place over the plate. (iii) The vortices, then, grow toward downstream and detach from the surface. Finally, they are disrupted significantly and a fully turbulent state is accomplished in the downstream region of the plate. The visualization experiments confirm that the above transition process actually occurs over the plate.

(2) The surface temperatures of the plate were visualized with a liquid-crystal sheet. The results were, then, compared with the analytical contours of the surface temperatures. The both results show identical temperature patterns. In particular, good agreement is obtained for the onset and the spanwise pitch of the low-temperature, streaky patterns. Moreover, the local heat transfer coefficients calculated from the analysis coincide well with those from experiment.

(3) In light of the above, it was confirmed that the present analysis predicts the actual transition phenomena quite satisfactory.

\section{References}

(1) Kitamura, K. and Kimura, F., Heat Transfer and Fluid Flow of Natural Convection over Horizontal, Heated Plate, Trans. Jpn. Soc. Mech. Eng., (in Japanese), Vol. 58, No. 556, B (1992), pp. $3715^{-}$ 3722.

(2) Kimura, F. and Kitamura, K., Heat Transfer and Fluid Flow of Natural Convection over Horizontal, Heated Plate (High-Rayleigh-Number Flow), Trans. Jpn. Soc. Mech. Eng., (in Japanese), Vol. 60, No. 570, B (1994), pp. 566-573.

(3) Kitamura, K. and Kimura, F., Heat Transfer and Fluid Flow of Natural Convection Adjacent to Upward-Facing Horizontal Plates, Int. J. Heat and Mass Transfer, Vol. 38, No. 17 (1995), pp. 3149-3159.

(4) Husar, R.B. and Sparrow, E.M., Patterns of Free Convection Flow Adjacent to Horizontal Heated Surface, Int. J. Heat and Mass Transfer, Vol. 11 (1968), pp. 1206-1212

( 5 ) Cheng, K.C. and Kim, Y.W., Flow Visualization Studies on Vortex Instability of Natural Convection Flow over Horizontal and Slightly Inclined Constant-Temperature Plates, Trans. ASME, J. Heat Transfer, Vol. 110, No. 3 (1988), pp. 608-615.

(6) Pera, L. and Gebhart, B., On the Stability of Natural Convection Boundary Layer Flow over Horizontal and Slightly Inclined Surfaces, Int. J. Heat and Mass Transfer, Vol. 16 (1973), pp. 11471162.

( 7 ) Chen T.S., Tien, H.C. and Armaly, B.F., Natural Convection on Horizontal, Inclined, and Vertical Plates with Variable Surface Temperature or Heat Flux, Int. J. Heat and Mass Transfer, Vol. 29, No. 19 (1986), pp. 1465-1478.

(8) Ishiguro, R., Abe, T. and Nagase, H., Natural Convection over Upward-Facing, Horizontal, Heated Plate (3rd Rep., Generation of Periodic Flow), Trans. Jpn. Soc. Mech. Eng., (in Japanese), Vol. 43, No. 375, B (1977), pp. 4223-4229.

(9) Lloyd, J.R. and Sparrow, E.M., On the Instability of Natural Convection Flow on Inclined Plates, J. Fluid Mech., Vol. 42, Pt. 3 (1970), pp. 465-470.

(10) Kitamura, K., Kami-iwa, F. and Misumi, T., Heat Transfer and Fluid Flow of Natural Convection around Large Horizontal Cylinders, Int. J. Heat Mass Transfer, Vol.42, No. 17 (1999), pp. 40934106 . 